\title{
Detection of left main coronary artery stenosis: utilization of transesophageal echocardiography in acute heart failure
}

\author{
T. G. V. Cherpanath • J. Baan • B. J. Bouma
}

Published online: 9 November 2011

(C) The Author(s) 2011. This article is published with open access at Springerlink.com

A previously healthy 80 -year-old male with a history of type II diabetes mellitus was referred to our emergency department because of severe dyspnoea. Physical examination revealed a diaphoretic man with a blood pressure of $105 / 65 \mathrm{mmHg}$, a low oxygen saturation of $85 \%$ and diffuse inspiratory crackles and rales on auscultation. The electrocardiogram showed a sinus tachycardia of 110 beats/min with a left bundle branch block. A chest X-ray of the lungs revealed pulmonary oedema. Arterial blood gas analysis indicated respiratory acidosis requiring intubation and ventilation. Immediate transoesophageal echocardiography (TEE) was performed to search for signs of suspected acute ischaemia. The echocardiogram pointed out a dilated left ventricle with severe hypokinesia of all wall segments. There was a moderate secondary mitral regurgitation due to the left ventricular dysfunction and annular dilatation. The aortic valve appeared normal without any signs of stenosis. The echocardiogram suggested the presence of chronic heart failure until colour Doppler analysis of the aortic valve revealed a mosaic flow pattern in the left main coronary artery suggesting a severe stenosis (Fig. 1a). Urgent cardiac catheterisation confirmed a pinpoint stenosis of the left main coronary artery (Fig. 1b). Subsequently, coronary artery bypass grafting was performed with maximum CK-MB of $55 \mu \mathrm{g} / \mathrm{L}$, and the patient recovered uneventfully.

T. G. V. Cherpanath $(\bowtie)$

Department of Intensive Care Medicine,

Academic Medical Centre,

Meibergdreef 9,

1105 AZ Amsterdam, the Netherlands

e-mail: t.g.cherpanath@amc.uva.nl

J. Baan · B. J. Bouma

Department of Cardiology, Academic Medical Centre,

Meibergdreef 9,

1105 AZ Amsterdam, the Netherlands

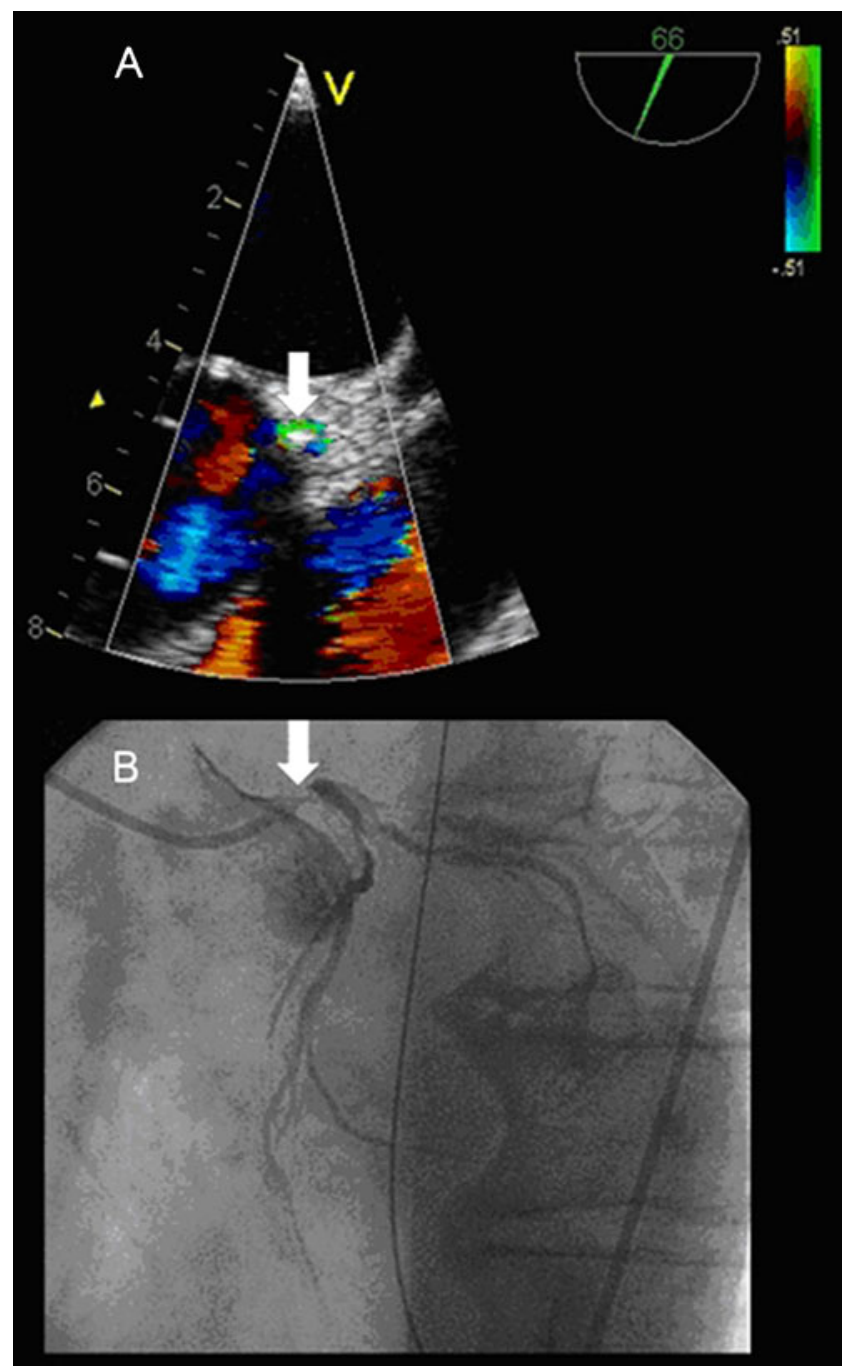

Fig. 1 a Colour Doppler analysis reveals mosaic flow in the left main coronary artery suggestive of a severe stenosis with the aortic valve showing no signs of obstruction; $\mathbf{b}$ In the LAO view the pinpoint left main coronary artery stenosis is confirmed 
Transthoracic echocardiography can adequately assess coronary flow in approximately half of patients [1]. Normally, the colour flow pattern in the coronary artery is consistent with laminar flow. Diastolic mosaic flow caused by focal flow acceleration indicates significant left main coronary artery stenosis in $85 \%$ of patients. Peak diastolic velocities greater than $1.5 \mathrm{~m} / \mathrm{s}$ are considered abnormal [1]. TEE can visualise haemodynamically significant stenosis of the left main as well as primarily proximal segments of the left anterior descending, circumflex and right coronary artery in the majority of patients [2]. Our case stresses the diagnostic power of a carefully performed TEE in haemodynamically unstable patients of unknown cause.
Open Access This article is distributed under the terms of the Creative Commons Attribution Noncommercial License which permits any noncommercial use, distribution, and reproduction in any medium, provided the original author(s) and source are credited.

\section{References}

1. Anjaneyulu A, Raghu K, Chandramukhi S, et al. Evaluation of left main coronary artery stenosis by transthoracic echocardiography. $\mathrm{J}$ Am Soc Echocardiogr. 2008;21:855-60.

2. Vrublevsky A, Boshchenko A, Karpov S. Diagnostics of main coronary artery stenoses and occlusions: multiplane transoesophageal doppler echocardiographic assessment. Eur J Echocardiogr. 2001;2:170-7. 